\title{
Clinical Usefulness and Predictability of Seoul National University Prostate Cancer Risk Calculator (SNU-PCRC)
}

\author{
Hyuk-Dal Jung', Hyeong Dong Yuk ${ }^{1}$, Ulanbek Balpukov ${ }^{1,2}$, Ja Hyeon $\mathrm{Ku}^{1}$, \\ Cheol Kwak', Hyeon Hoe Kim', Chang Wook Jeong ${ }^{1}$ \\ ${ }^{1}$ Department of Urology, Seoul National University Hospital, Seoul, Korea \\ ${ }^{2}$ Department of Urology and Andrology, JSK Medical University Astana, Astana, Kazakhstan
}

\begin{abstract}
Purpose: To evaluate the clinical usefulness of the Seoul National University Prostate Cancer Risk Calculator (SNU-PCRC) to reduce unnecessary prostate biopsy and to increase the detection rate of high-risk cancer. Materials and Methods: We retrospectively analyzed 546 patients who underwent prostate biopsy between 2014 and 2016. The subjects were divided into 2 groups based on the type of risk calculator used: conventional and SNU-PCRC group. In the SNU-PCRC group, prostate biopsy was recommended when the probability of SNU-PCRC was more than $30 \%$.

Results: The SNU-PCRC group had significantly smaller prostate volume $(p=0.010)$ and significantly more digital rectal examination and transrectal ultrasonography (TRUS) abnormalities ( $p=0.011$ and $p=0.010$, respectively). Overall detection $(71.9 \%$ vs. $32.1 \%)$ and high-risk cancer detection rates $(40.6 \%$ vs. $19.3 \%)$ were significantly higher in the gray zone (prostate-specific antigen=4-10 ng/mL) $(p<0.001$ and $p=0.006)$. The group with prostate cancer risk $\geq 30 \%$ on the SNU-PCRC compared to $<30 \%$ group, overall detection rate of $72.3 \%$ versus $30.2 \%$ and high-risk detection rate of $60.6 \%$ versus $18.3 \%$ were significantly different $(p<0.001$ and $p<0.001)$. Applying the SNU-PCRC to the conventional group could avoid unnecessary prostate biopsy in $50.6 \%$.

Conclusions: SNU-PCRC is clinically useful to reduce unnecessary prostate biopsy and increase overall detection rate and high-risk cancer detection rate. (Korean J Urol Oncol 2020;18:215-221)
\end{abstract}

Key Words: Cancer detection - Prostate biopsy - Prostate cancer · Risk calculator

\section{INTRODUCTION}

Prostate cancer $(\mathrm{PCa})$ is the second most common cancer

Received September 24, 2020, Revised November 12, 2020,

Accepted November 16, 2020

Corresponding Author: Chang Wook Jeong

Department of Urology, Seoul National University Hospital, 101

Daehak-ro, Jongno-gu, Seoul 03080, Korea

E-mail: drboss@korea.com

Tel: +82-2-2072-3899, Fax: +82-2-742-4665

ORCID: https://orcid.org/0000-0002-2200-5019

- This study was supported by the National R\&D Program for Cancer Control, Ministry of Health and Welfare, Republic of Korea (grant numbers: HA17C0039). in men worldwide. It is the sixth cause of cancer related deaths. ${ }^{1} \mathrm{PCa}$ is common, and one of the major reasons is that the start of the use of a screening tool called prostate-specific antigen (PSA). ${ }^{2}$ However, PCa screening based on these PSA values lacks specificity. ${ }^{3}$ The lack of specificity leads to overdiagnosis and excessive prostate biopsy. ${ }^{4}$ Prostate biopsy may be accompanied by morbidity and mortality as well as socioeconomic costs and discomfort. ${ }^{5}$ Due to the associated mortality and morbidity, it is difficult and crucial to determine if biopsy is indicated in practice. ${ }^{6}$ To assist in this decision, several studies have examined various nomograms and calculators. ${ }^{7-11}$ In this regard, we devel-

(i) (8) This is an Open Access article distributed under the terms of the Creative Commons Attribution Non-Commercial License (http://creativecommons.org/licenses/by-nc/4.0/) which permits unrestricted non-commercial use, distribution, and reproduction in any medium, provided the original work is properly cited. 2020 (C) Copyright The Korean Urological Oncology Society and The Korean Prostate Society. All Rights Reserved. 
oped and announced the "Seoul National University Prostate Cancer Risk Calculator (SNU-PCRC)" mobile application in 2014. In our previous study, we compared SNU-PCRC with the European Randomized Study of Screening for Prostrate Cancer Risk Calculator (ERSPCRC) and the Prostate Cancer Prevention Trial Risk Calculator (PCPT-RC) calculators. Our calculator has been designed to fit the characteristics of Korean populations and has higher prediction accuracy than other calculators. ${ }^{12}$

We analyzed the actual clinical use of the SNU-PCRC for approximately 2.5 years from 2014 and explore its usefulness in this study.

\section{MATERIALS AND METHODS}

\section{Seoul National University Prostate Cancer Risk Calculator}

The SNU-PCRC was developed on the basis of data from 5,278 people who underwent prostate biopsy of more than 12-core between 2003 and 2010 in Seoul National University Bundang Hospital. PSA level, patients age, prostate size, abnormality on digital rectal examination or transrectal ultrasonography (TRUS) are the variables that constitute the calculator. ${ }^{12}$ The nomogram predicting the probability of PCa was developed using this final multivariate logistic regression model. The multivariate model was constructed based on a significant variable with a p-value of 0.05 or less in the univariate analysis. In the model, PSA levels and prostate size were standardized by $\log$ transformations. The logit of SNU-PCRC was calculated as $(0.21)+$ $(0.071 \times$ Age $)+(2.379 \times \log 10 \mathrm{PSA})-(4.775 \times \log 10 \mathrm{Vol})+(0.544 \times$ TRUS $)+(0.397 \times D R E)$. Head-to-head comparison was performed with the European Randomized Study of Screening for Prostate Cancer Risk Calculator (ERSPC-RC) and the PCPT-RC for the same cohort. The prediction accuracy of SNU-PCRC (area under the curve [AUC], 0.811; 95\% confidence interval $[\mathrm{CI}], 0.786^{-0.833)}$ was better than that of ERSPC-RC (AUC, 0.768; 95\% CI, 0.742-0.792; p<0.001) and PCPT-RC (AUC, 0.704; 95\% CI, 0.676-0.731; p<0.001).

The threshold probability of SNU-PCRC was chosen as the percentage of the highest sensitivity and specificity in the receiver operating characteristic (ROC) curve and compared with the traditional PSA cutoff $>4 \mathrm{ng} / \mathrm{mL}$. In the previous study, the threshold probability of SNU-PCRC was
$30 \%$, sensitivity was $76.3 \%$, specificity was $61.3 \%$, false positive rate was $56.3 \%$ and false negative rate was $85.7 \%$. The sensitivity, specificity, positive predictive value, and negative predictive value of PSA $4 \mathrm{ng} / \mathrm{mL}$ were $86.8 \%$, $26.9 \%, 41.6 \%$, and $77.3 \%$ respectively.

\section{Study Sample}

The study was approved by the Institutional Review Board of Seoul National University Hospital (IRB No: 1710-077-893). Content and protocol associated with this study followed the Declaration of Helsinki guidelines. We retrospectively analyzed prospectively collected data of patients that visited Seoul National University Hospital from January 2014 through August 2016. All patients underwent transrectal ultrasound prostate 12-core systematic biopsy and patients with PSA $>100 \mathrm{ng} / \mathrm{mL}$ were excluded. Patients under active surveillance with PCa diagnosis were excluded. Finally, 546 patients were included.

\section{Study Design}

We divided patients into 2 groups: a group that underwent PCa risk assessment with the SNU-PCRC calculator and a group that did not (conventional group). The conventional group was a group that performed biopsy based on PSA levels alone. Prostate biopsy was routinely conducted on 12 cores with conventional guided TRUS biopsy and conducted by trained professional doctors. Approximately $50 \%$ of the biopsies were conducted by urologists and $50 \%$ by radiologists. For accuracy of diagnosis, tissue obtained by TRUS biopsy was reviewed by 2 experienced genitourinary pathologists. In SNU-PCRC group, prostate biopsy was recommended when probability of $\mathrm{PCa}$ according to the SNU-PCRC was more than $30 \%$. In the non-SNU-PCRC group, PSA screening was used as a reference for prostate biopsy. Pre-operational demographics and post biopsy outcomes include various data such as PSA, DRE findings, TRUS abnormal findings, volume and prostate biopsy core number, number of positive cores, Gleason score, overall detection rate, and high-risk detection rate. High-risk PCa was defined as a cancer with a Gleason score of 7 or higher. Abnormal findings on TRUS biopsy include hypoechoic lesions and hyperechoic lesions, as well as abnormal flow lesions in color Doppler images. 


\section{Statistical Analysis}

Statistical analysis for continuous variables, mean, and standard deviation are shown. Variables of categories are indicated by frequencies of events (\%). Primary endpoint of the study was overall detection rate. Secondary endpoint was high-risk PCa detection rate. Outcomes were analyzed using analysis of variance, $\mathrm{t}$-paired test, and crossover analysis. Specificity, sensitivity, positive predictive value, and negative predictive value were analyzed using ROC curve.

\section{RESULTS}

\section{Clinical Characteristics of Previous Prostate Biopsy of Patients}

Table 1 reveals clinical characteristics of 546 patients. There was no significant difference in PSA averages between the conventional and the SNU-PCRC group. However, the SNU-PCRC group had more suspicious DRE findings and abnormal TRUS findings than the conventional group ( $\mathrm{p}=0.011$ and $\mathrm{p}=0.002$ ). Prostate volume was relatively small in the SNU-PCRC group $(\mathrm{p}=0.010)$.

\section{Comparison of Prostate Biopsy Outcomes Between the SNU-PCRC Group and the Conventional Group}

Table 2 reveals the comparison of outcomes after prostate biopsy between the 2 groups. Although not significant, the prostate volume was larger in the conventional group. Overall detection and high-risk PCa detection were significantly higher in the SNU-PCRC group $(\mathrm{p}=0.001$ and $\mathrm{p}=0.036$ ). We subdivided overall detection and high-risk detection PCa into 3 groups according to PSA: PSA $<4$ $\mathrm{ng} / \mathrm{mL}$, PSA 4-10 ng/mL, and PSA >10 ng/mL. Among them, the SNU-PCRC group revealed higher overall and high-risk detection rate especially in 4-10 sections called gray zone.

Fig. 1 reveals the trend of detection rates overall, in the SNU-PCRC, and conventional populations at the 3 sections below PSA 4, PSA 4-10, and above PSA 10. Most of them have a higher detection rate towards PSA>10 ng/mL. However, based on SNU-PCRC, overall detection in the gray zone (PSA $4-10 \mathrm{ng} / \mathrm{mL}$ ) is higher than in the PSA $>10$ $\mathrm{ng} / \mathrm{mL}$ group.

In 18 patients $(6.6 \%)$ who underwent TRUS biopsy scoring with $\mathrm{PSA}<4 \mathrm{ng} / \mathrm{mL}$ in the SNU-PCRC group, the mean age was $67.2 \pm 7.0$ years, PSA was $2.4 \pm 1.3 \mathrm{ng} / \mathrm{mL}$, and the prostate volume was $26.0 \pm 9.7 \mathrm{~mL}$. The overall rate of detection and the proportion of high-risk cancer was $44.4 \%$. Patients who underwent biopsy with PSA $\leq 4 \mathrm{ng} / \mathrm{mL}$ were patients with DRE and TRUS abnormalities, or other diseases, and were suspected of PCa based on radiology findings and follow-up examination.

\section{SNU-PCRC Application in Conventional Group Patients}

SNU-PCRC was applied to the patients in the conven-

Table 1. Patient characteristics before prostate biopsy

\begin{tabular}{lcrc}
\hline \multicolumn{1}{c}{ Variable } & Conventional group $(\mathrm{n}=432)$ & SNU-PCRC group $(\mathrm{n}=114)$ & $\mathrm{p}$-value \\
\hline Age $(\mathrm{yr})$ & $67.1 \pm 8.1$ & $68.7 \pm 8.8$ & 0.176 \\
BMI $\left(\mathrm{kg} / \mathrm{m}^{2}\right)$ & $24.22 \pm 3.20$ & $24.41 \pm 3.12$ & 0.668 \\
PSA $(\mathrm{ng} / \mathrm{mL})$ & $10.1 \pm 12.32$ & $12.0 \pm 15.7$ & 0.395 \\
DRE suspicious finding & $86(19.9)$ & $32(26.3)$ & 0.011 \\
Previous TRUS abnormal findings & $179(41.4)$ & $72(63.2)$ & 0.002 \\
Previous TRUS volume (mL) & $55.6 \pm 26.9$ & $47.4 \pm 21.4$ & 0.010 \\
Total biopsy core number & & & 0.119 \\
12 & $343(79.4)$ & $80(70.2)$ & $34(29.8)$ \\
13 & $71(16.4)$ & $0(0)$ & \\
14 & $18(4.2)$ & & \\
\hline
\end{tabular}

Values are presented as mean \pm standard deviation or number (\%).

SNU-PCRC: Seoul National University Prostate Cancer Risk Calculator, BMI: body mass index, PSA: prostate specific antigen, DRE: digital rectal examination, TRUS: transrectal ultrasonography. 
Table 2. Results of prostate biopsy according to SNU-PCRC application

\begin{tabular}{lccc}
\hline \multicolumn{1}{c}{ Variable } & Conventional group $(\mathrm{N}=432)$ & SNU-PCRC group (N=114) & $\mathrm{p}$-value \\
\hline Overall detection rate & $176(40.7)$ & $74(64.9)$ & 0.001 \\
High risk prostate cancer detection rate & $130(30.1)$ & $50(43.9)$ & 0.036 \\
Positive core & $1.9 \pm 3.1$ & $2.7 \pm 3.4$ & 0.114 \\
Gleason score & & & 0.789 \\
6 & $44(25.0)$ & $22(29.7)$ & \\
$7(3+4)$ & $44(25.0)$ & $12(16.2)$ \\
$7(4+3)$ & $33(18.8)$ & $16(21.6)$ \\
8 & $31(17.6)$ & $8(10.8)$ \\
9 & $19(10.8)$ & $0(0)$ \\
\hline
\end{tabular}

Values are presented as number (\%) or mean \pm standard deviation.

SNU-PCRC: Seoul National University Prostate Cancer Risk Calculator.

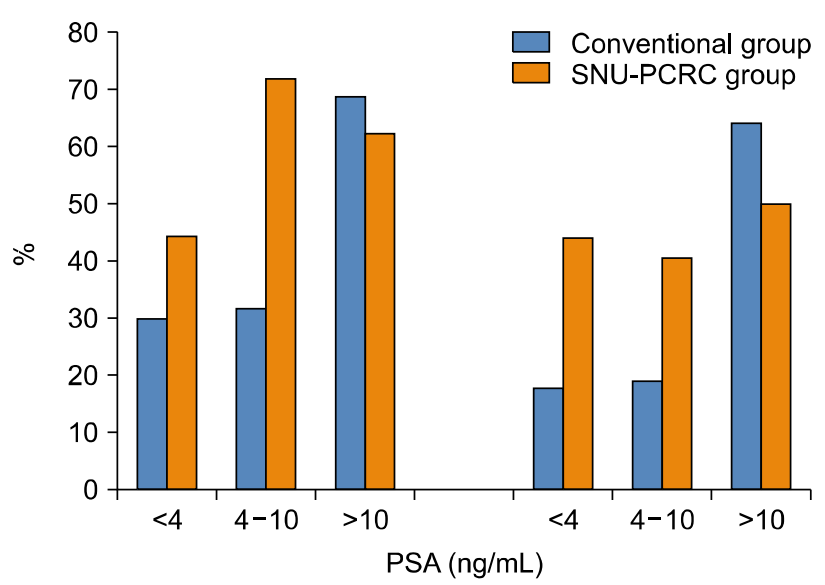

Fig. 1. Overall prostate cancer detection rate and high- risk prostate cancer detection rate according to prostate-specific antigen (PSA) interval. SNU-PCRC: Seoul National University Prostate Cancer Risk Calculator.

tional group. The conventional group was then divided into 2 groups: those with a SNU-PCRC probability score of $>30 \%$ and those with a probability score of $<30 \%$. In patients with a probability score $>30 \%$, the overall detection rate and the high-risk cancer detection rate were $72.8 \%$ and $61.8 \%$, respectively. On the other hand in patients with a probability score of $<30 \%$, the overall detection rate and the high-risk cancer detection rate were $26 \%$ and $15.5 \%$, respectively.

\section{DISCUSSION}

When considering prostate biopsy through PSA screening tests, there are many ambiguities about whether to conduct a biopsy or not. PSA-based screening contributed significantly to PCa-specific mortality reduction. ${ }^{13,14}$ However, PSA-based screening leads to unnecessary invasive tests and overdiagnosis. ${ }^{15}$ It can cause complications due to biopsy such as infections. The is also the risk of infection with multidrug resistant bacteria. ${ }^{16,17}$ Feliciano et al. ${ }^{16}$ reported that the incidence of resistant bacteria increased in patients that developed infection after prostate biopsy. Otrock et al. ${ }^{18}$ reported that fluoroquinolone resistant Escherichia coli was detected in $50 \%$ of patients admitted for urinary tract infection (UTI) after prostate biopsy. Additionally, several studies have reported cases of sepsis and disseminated intravascular coagulation deaths due to antibiotic resistant bacteria after prostate biopsy. ${ }^{19-21}$ Although prophylactic antibiotics were used, prostate biopsy could cause complications such as UTI. Additionally, antibiotic resistant bacteria may be acquired and leading to sepsis and death. After prostate biopsy, infection complications will may result in an increase in treatment costs for the patient even if the patient becomes asymptomatic and healthy. Unnecessary biopsy causes inspection costs and this can be a great burden on patients. For these reasons, the decision to perform a prostate biopsy must be taken with caution. SNU-PCRC facilitates more effective decision-making for invasive procedures in such situations. The SNU-PCRC calculates cancer risk using age, PSA, suspicious findings in digital rectal examination, prostate volume, and abnormal findings in TRUS. $^{22}$ In our study, the SNU-PCRC group revealed a 1.5 times higher overall detection rate or high-risk $\mathrm{PCa}$ detection rate than the conventional group (Table 2). 
Additionally, we observed a difference of detection rate of more than 2 times in the gray zone when PSA 4-10 $\mathrm{ng} / \mathrm{mL}$, the value that we are concerned about whether biopsy is conducted (Fig. 1). The use of SNU-PCRC allows improved patient selection and increased detection rate. The selection of these patients and increase in cancer detection rates have an advantage of reducing the cost of an annual PCa screening. Compared to PSA alone, it does not add much cost, but can increase efficiency.

The overall detection rate and the proportion of high-risk PCa was $44.4 \%$ when the SNU-PCRC was used for cases scoring $\mathrm{PSA}<4 \mathrm{ng} / \mathrm{mL}$. Patients with a high-risk of PCa with $\mathrm{PSA}<4 \mathrm{ng} / \mathrm{mL}$ were characterized by a small volume and abnormal ultrasonographic findings. The mean prostate volume was $26.0 \pm 9.7 \mathrm{~mL}$. We found that $77 \%$ of the cases showed abnormal findings in TRUS, and $22.2 \%$ had suspected DRE findings. Prostate volume is an important component of SNU-PCRC. Although PSA tends to increase with the prostate volume, the detection rate of cancer in prostate biopsy tends to increase with decreased prostate volume because the smaller the tumor, the higher the probability of a biopsy core contacting the tumor. ${ }^{23}$ In other predictive nomograms, prostate volume was an important parameter and showed a tendency to decrease with increasing prostate volume. ${ }^{24}$ PSA density (PSAD), which is the ratio between the absolute PSA value and the prostate volume, is used to classify PSA based on prostate volume measured by ultrasound. The lower the prostate volume, the higher the PSAD value. The PSAD of benign prostatic hyperplasia is low and the PSAD of PCa is high. ${ }^{25}$
As can be seen in Table 3, prostate biopsy might be avoided in approximately $74 \%$ of patients when SNUPCRC was applied to the conventional group. When SNUPCRC was applied to the conventional group, $68.5 \%$ patients had $<30 \%$ probability of PCa considering prostate biopsy in SNU-PCRC. Of these, 26\% had cancer. Conversely, $74 \%$ of patients were cancer-free. If SNU-PCRC was applied, 219 patients, $50.6 \%$ of the total conventional group patients, could have avoided an unnecessary biopsy. Considering only high-risk $\mathrm{PCa}$, unnecessary biopsies were reduced in $57.8 \%$ of the total conventional group. By using SNU-PCRC, we can avoid unnecessary use of prostate biopsy that can cause complications. Reducing unnecessary biopsy reduces patient care costs. Ninety-four patients were observed during the same period after SNU-PCRC was applied, accounting for $40 \%$ of SNU-PCRC patients. The mean age of these patients was $68.4 \pm 8.3$ years, PSA was $4.47 \pm 1.70 \mathrm{ng} / \mathrm{mL}$, mean prostate volume was $55.36 \pm 25.79$ $\mathrm{mL}, 7.44 \%$ of patients had abnormal TRUS findings, and $2.12 \%$ of patients had DRE abnormalities.

SNU-PCRC is useful and excellent, but there are a few shortcomings. Since the sensitivity and specificity of SNU-PCRC are not $100 \%$, a positive diagnosis may be missed with a biopsy even if the patient may have PCa. Recently, dynamic contrast-enhanced, diffusion-weighted imaging, T2-weighted combined multiparametric magnetic resonance imaging (MRI), and magnetic resonance fusion biopsy have recently been beneficial in diagnosing $\mathrm{PCa}^{25,26}$ Techniques for detecting PCa using MRI have been improved by application of apparent diffusion coefficient val-

Table 3. Result of applying SNU-PCRC to convention group

\begin{tabular}{lccc}
\hline \multicolumn{1}{c}{ Variable } & SNU-PCRC $<30 \%$ & SNU-PCRC $\geq 30 \%$ & p-value \\
\hline Number & 296 & 136 & $<0.001$ \\
Overall detection rate & $77(26.0)$ & $99(72.8)$ & $<0.001$ \\
High risk prostate cancer detection rate & $46(15.5)$ & $84(61.8)$ & $<0.001$ \\
Gleason score & & & \\
6 & $31(40.3)$ & $14(14.1)$ & \\
$7(3+4)$ & $24(31.2)$ & $18(18.2)$ & \\
$7(4+3)$ & $15(19.5)$ & $20(20.2)$ & \\
8 & $4(5.2)$ & $27(27.3)$ & \\
9 & $2(2.6)$ & $17(17.2)$ & \\
10 & $1(1.3)$ & $2(2.0)$ & \\
\hline
\end{tabular}

Values are presented as number (\%).

SNU-PCRC: Seoul National University Prostate Cancer Risk Calculator. 
ue and Prostate Imaging Reporting and Data System (PIRADS) scoring system. ${ }^{27}$ It is applied to various AS protocols. ${ }^{28}$ We think that this MRI-related scoring system could improve the sensitivity and specificity by applying PIRADS scoring system as an element to SNU-PCRC. Our study has several limitations. First, this study is a retrospective study and cannot be free from selection bias. Second, the SNU-PCRC group may have been selected as a group with a slightly higher PCa risk compared to the control group due to the application of the PCa risk calculator. There may be a bias in the underlying nature of the patient population.

\section{CONCLUSIONS}

When SNU-PCRC is applied, the PCa detection rate is higher than when PSA, DRE, TRUS abnormality, and age, especially in the gray zone between PSA 4-10 ng/mL are considered as predictors of risk. It can effectively assist in the determination of invasive procedures in patients with PSA 4-10 $\mathrm{ng} / \mathrm{mL}$. It can facilitate in reducing unnecessary biopsy and reducing socioeconomic costs.

\section{CONFLICT OF INTEREST}

The authors claim no conflicts of interest.

\section{REFERENCES}

1. Torre LA, Bray F, Siegel RL, Ferlay J, Lortet-Tieulent J, Jemal A. Global cancer statistics, 2012. CA Cancer J Clin 2015;65:87-108.

2. Stamey TA, Yang N, Hay AR, Mcneal JE, Freiha FS, Redwine E. Prostate-specific antigen as a serum marker for adenocarcinoma of the prostate. New Engl J Med 1987;317:909-16.

3. van Vugt HA, Roobol MJ, Kranse R, Maattanen L, Finne $\mathrm{P}$, Hugosson $\mathrm{J}$, et al. Prediction of prostate cancer in unscreened men: external validation of a risk calculator. Eur J Cancer 2011;47:903-9.

4. Bokhorst LP, Bangma CH, van Leenders GJ, Lous JJ, Moss SM, Schroder FH, et al. Prostate-specific antigenbased prostate cancer screening: reduction of prostate cancer mortality after correction for nonattendance and contamination in the Rotterdam section of the European Randomized Study of Screening for Prostate Cancer. Eur Urol 2014;65:329-36.
5. Pinsky PF, Parnes HL, Andriole G. Mortality and complications after prostate biopsy in the Prostate, Lung, Colorectal and Ovarian Cancer Screening (PLCO) trial. BJU Int 2014;113:254-59.

6. Kibel AS. To biopsy or not to biopsy: minimizing the risk of prostate needle biopsy. J Urol 2013;189:796-7.

7. Briganti A. Editorial comment on: Prostate cancer gene 3 (PCA3): development and internal validation of a novel biopsy nomogram. Eur Urol 2009;56:667-8.

8. Huang Y, Cheng G, Liu B, Shao P, Qin C, Li J, et al. A prostate biopsy strategy based on a new clinical nomogram reduces the number of biopsy cores required in highrisk patients. BMC Urol 2014;14:8.

9. Kranse R, Roobol M, Schroder FH. A graphical device to represent the outcomes of a logistic regression analysis. Prostate 2008;68:1674-80.

10. Remzi M, Fong YK, Dobrovits M, Anagnostou T, Seitz $\mathrm{C}$, Waldert $\mathrm{M}$, et al. The Vienna nomogram: validation of a novel biopsy strategy defining the optimal number of cores based on patient age and total prostate volume. J Urol 2005;174(4 Pt 1):1256-60; discussion 1260-1; author reply 1261 .

11. Thompson IM, Ankerst DP, Chi C, Goodman PJ, Tangen CM, Lucia MS, et al. Assessing prostate cancer risk: results from the Prostate Cancer Prevention Trial. J Natl Cancer Inst 2006;98:529-34.

12. Jeong CW, Lee S, Jung JW, Lee BK, Jeong SJ, Hong SK, et al. Mobile application-based Seoul National University Prostate Cancer Risk Calculator: development, validation, and comparative analysis with two Western risk calculators in Korean men. PLoS One 2014;9:e94441.

13. Schroder FH, Hugosson J, Roobol MJ, Tammela TL, Zappa M, Nelen V, et al. Screening and prostate cancer mortality: results of the European Randomised Study of Screening for Prostate Cancer (ERSPC) at 13 years of follow-up. Lancet 2014;384:2027-35.

14. Slomski A. USPSTF finds little evidence to support advising PSA screening in any man. JAMA 2011;306:2549-51.

15. Loeb S, Carter HB, Berndt SI, Ricker W, Schaeffer EM. Complications after prostate biopsy: data from SEERMedicare. J Urol 2011;186:1830-4.

16. Feliciano J, Teper E, Ferrandino M, Macchia RJ, Blank W, Grunberger I, et al. The incidence of fluoroquinolone resistant infections after prostate biopsy--are fluoroquinolones still effective prophylaxis? J Urol 2008;179:952-5; discussion 955.

17. Loeb S, van den Heuvel S, Zhu X, Bangma CH, Schroder FH, Roobol MJ. Infectious complications and hospital admissions after prostate biopsy in a European randomized trial. Eur Urol 2012;61:1110-4.

18. Otrock ZK, Oghlakian GO, Salamoun MM, Haddad M, Bizri AR. Incidence of urinary tract infection following 
transrectal ultrasound guided prostate biopsy at a tertiary-care medical center in Lebanon. Infect Control Hosp Epidemiol 2004;25:873-7.

19. Dan M, Yair Y, Samosav A, Gottesman T, Yossepowitch $\mathrm{O}$, Harari-Schwartz $\mathrm{O}$, et al. Escherichia coli isolates from patients with bacteremic urinary tract infection are genetically distinct from those derived from sepsis following prostate transrectal biopsy. Int J Med Microbiol 2015;305: 464-8.

20. Miura T, Tanaka K, Shigemura K, Nakano Y, Takenaka A, Fujisawa M. Levofloxacin resistant Escherichia coli sepsis following an ultrasound-guided transrectal prostate biopsy: report of four cases and review of the literature. Int J Urol 2008;15:457-9.

21. Urban JA. Cost analysis of surgical site infections. Surg Infect (Larchmt) 2006;7 Suppl 1:S19-22.

22. Miller AB. New data on prostate-cancer mortality after PSA screening. N Engl J Med 2012;366:1047-8.

23. Kawakami S, Numao N, Okubo Y, Koga F, Yamamoto S, Saito K, et al. Development, validation, and head-to-head comparison of logistic regression-based nomograms and artificial neural network models predicting prostate cancer on initial extended biopsy. Eur Urol 2008;54:601-11.

24. Chun FK, Briganti A, Graefen M, Montorsi F, Porter C, Scattoni $\mathrm{V}$, et al. Development and external validation of an extended 10-core biopsy nomogram. Eur Urol 2007;52: 436-44.

25. Walz J. Words of Wisdom. Re: Comparison of MR/ultrasound fusion-guided biopsy with ultrasound-guided biopsy for the diagnosis of prostate cancer. Eur Urol 2016;69:7567.

26. Lin WC, Westphalen AC, Silva GE, Chodraui Filho S, Reis RB, Muglia VF. Comparison of PI-RADS 2, ADC histogram-derived parameters, and their combination for the diagnosis of peripheral zone prostate cancer. Abdom Radiol (NY) 2016;41:2209-17.

27. Soloway MS, Soloway CT, Eldefrawy A, Acosta K, Kava B, Manoharan M. Careful selection and close monitoring of low-risk prostate cancer patients on active surveillance minimizes the need for treatment. Eur Urol 2010;58:831-5.

28. van den Bergh RC, Roemeling S, Roobol MJ, Roobol W, Schroder FH, Bangma CH. Prospective validation of active surveillance in prostate cancer: the PRIAS study. Eur Urol 2007;52:1560-3. 